\title{
KARMAN VORTEX VELOCIMETER WITH A GENERATOR OF VERY SMALL DIAMETER
}

\author{
Susumu SAITO*, Satoshi TAKAGI**, Tami SAWADA*** \\ *School of Management, Science University of Tokyo \\ 346 Shimokiyoku Kuki-shi Saitama \\ **Department of Mechanical Engineering, Science University of Tokyo \\ $* * *$ Ricoh Co,
}

\begin{abstract}
Karman Vortex velocimeter which is applicable to liquid and gas was developed in this study. In this case, the Karman vortex generator of a very small diameter $\phi 0.1 \sim 0.7)$ is set perpendicular to the flow and a parallel laser beam $(\dot{\phi}$ 1) from laser diode passes through downstream of the generator. This beam is refracted by the density change resulted from the Karman vortex. The refracted light is detected by the photo-diode and the frequency is measured with FFT. The generator is heated to strengthen the magnitude of the signal. Three generators of three different diameter $\phi 0.3,0.5,0.7)$ are used to measure the velocities of three different points simultaneously and nine generators of same diameter ( $\dot{0} 0.1$ ) are used to switch the measuring point instantly by switching the electrical charge to the generators.
\end{abstract}

KEYWORDS: velocimeter, Karman vortex, Laser diode,

\section{INTRODUCTION}

So far, various kind of velocimeters have been developed. Among them laser doppler velocimeter has excellent characteristics such as noninvasive measurement and no calibration.

In this study, the velocimeter which measures the Karman vortex frequency at thedownstream of the vortex generator has been developed and reported[1][2]. Although this method is not noninvasive, metal wire ( $\mathrm{Ni}-\mathrm{Cr}$ ) of very small diameter $(\phi 0.1 \sim \phi 0.3)$ is used and therefore the influence to the flow is minute. To detect the Karman vortex, laser diode is used which is cheap and optically easy to handle. The metal wire is applied an electrical charge to strengthen the signal.

The previous reports[1][2] showed the results concerning single generator. In this report, three generators of different diameter( $\phi$ $0.3,0.5,0.7)$ were used to investigate the possibility of simultaneous measurement of three points and nine generators of same diameter( $\phi 0.1)$ were tested to carry out instantaneous switching of measuring point by switching the electrical charge.

\section{PRINCIPLE OF MEASUREMENT}

Fig. 1 shows the principle of measurement. Small parallel laser light passes through about 
three times downstream of the diameter of vortex generator.

The laser light is refracted by the density change of the vortex and the refracted light is detected by photo-diode and the detected signals are analyzed by FFT. To strengthen the signal, electrical current is applied to the wire to heat it.

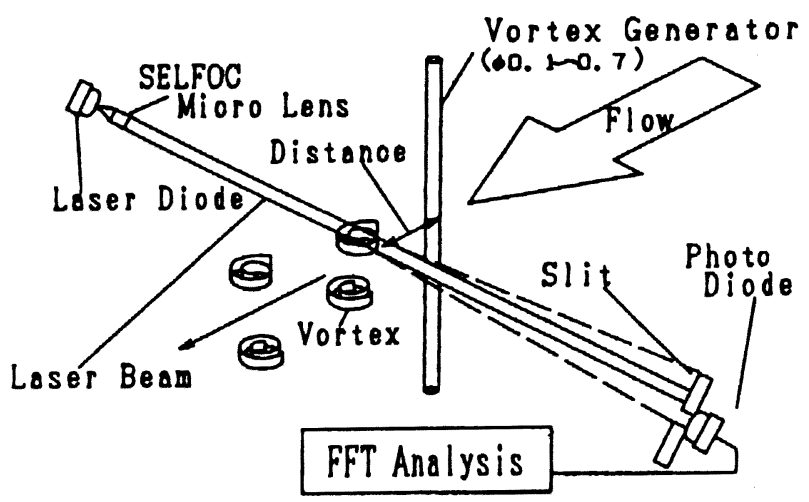

Fig. I Schematic diagram

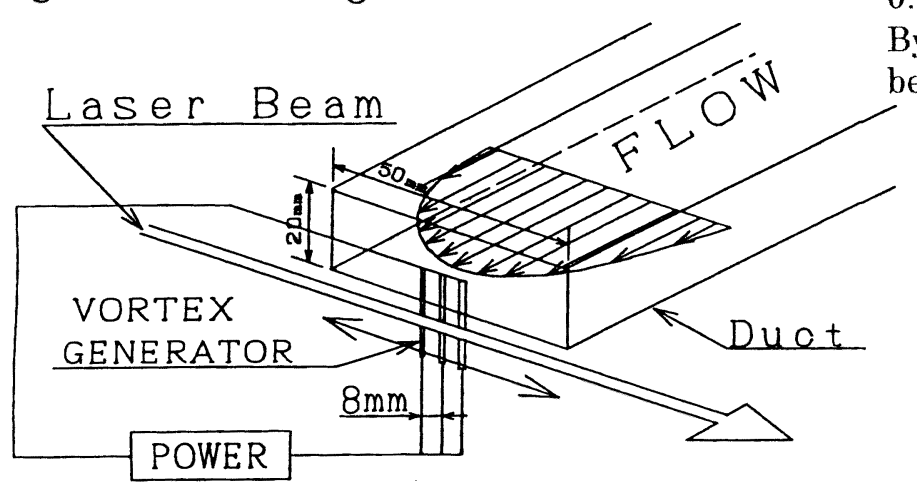

(a) Three generators $(\phi 0.3,0.5,0.7)$

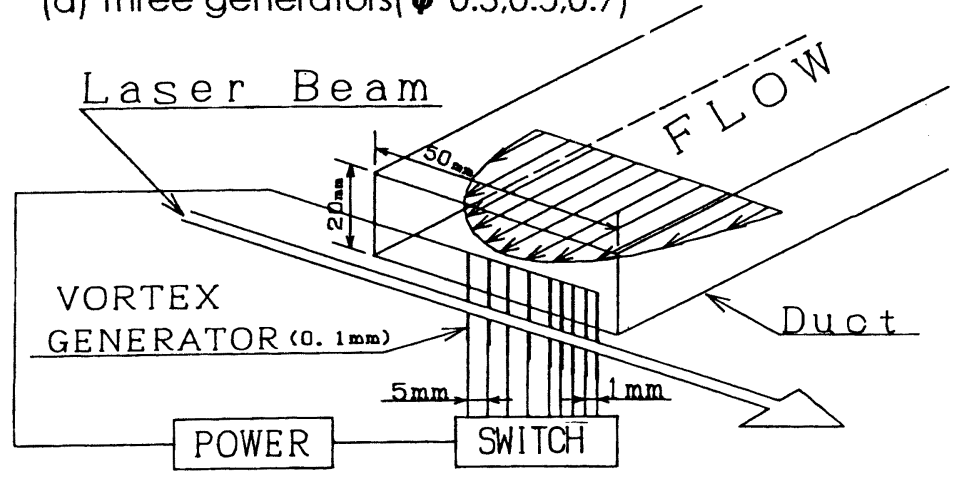

(b) Nine generators $(\$ 0.1)$

Fig.2 Experimental apparatus

\section{EXPERIMENTATION}

The laser light (laser diode ,780nm,20mw) is collimated by SELFOC micro-lens to $\phi 1 \mathrm{~mm}$ parallel light. The channel is $20 \mathrm{~mm} \times 50 \mathrm{~mm}$ cross section .

The experimentations are done for (A) the vortex generators of different diameter and (B) the vortex generators of same size as stated below.

(A) The vortex generators of different diameter

Fig.2 (a) shows the example of three generators of different diameter set at the exit of the channel. The different frequencies for each generator are detected simultaneously by one laser beam. By this method, the simultaneous measurement of two or three different points can be done.

In this case, the generators of the diameter of $\phi$ $0.3 \mathrm{~mm}, \phi 0.5 \mathrm{~mm}, \phi 0.7 \mathrm{~mm}$ were employed.

By moving these generators along the laser beam with keeping constant distance of the adjoining generators, the velocity profile can be measured.

(B) The vortex generators of same size As stated in the principle, the electrical current is applied to the metal wire to make the detection easier because the density change by the Karman vortex becomes large. This means that the signal becomes small if the current is not applied. This method is using this phenomena.

Nine vortex generators of same size ( $\phi$ $0.1 \mathrm{~mm}$ ) are set in parallel at the exit of the channel as shown in Fig.2 (b). The electric power is applied to only one generator in this case. The current can be switched instantly and the measuring point is able to be changed quickly. In this case,the velocity profile can be measured without moving the vortex generators and the laser beam. 


\section{RESULTS AND CONSIDERATION}

Fig.3 shows the relations between the frequencies and velocity of air in case of single generator of various diameter.

From this figure, it is clear that the velocity and the frequency is almost linear and the velocity of over $200 \mathrm{~m} / \mathrm{s}$ can be measured.

(A) The vortex generators of different diameter

Fig.4 shows the power spectrums of two generators $(\phi 0.3, \phi 0.5)$ at various point keeping the distance between the generators constant $(13 \mathrm{~mm})$. In this case, the generators of $\phi 0.5$ is set at the center of the channel as initial set.

The two peeks of the spectrums overlap each other as the distance from the center becomes large.

On the other hand, Fig.5 shows the case that the generator of $\phi 0.3$ is set at center as initial set.

In this case, the discrimination of the peaks is distinct.

Fig.6 shows the velocity profile which was measured by the method of Fig.5. Almost same

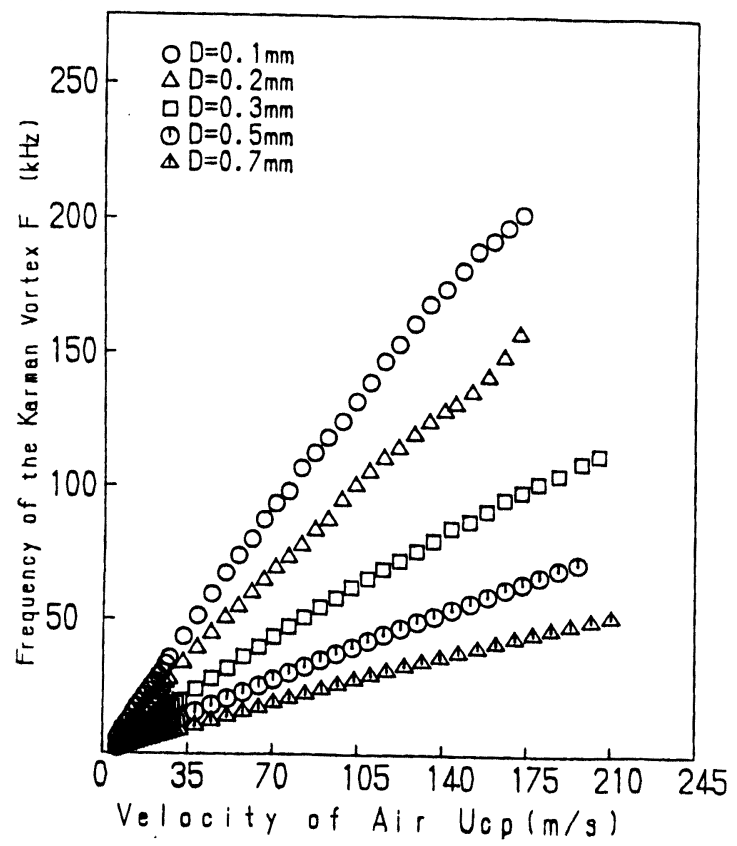

Fig.3 Frequency of the Karman vortex

vs. Velocity of air result was obtained as compared with that of Pitot-tube.

Fig. 7 shows the case of three generators $(\phi 0.3$, $\phi 0.5, \phi 0.7)$. The generator of $\phi 0.3$ is set on the center at initial set and the generator of $\phi$ 0.5 is set $8 \mathrm{~mm}$ apart from the generator of $\phi 0.3$ and the generator of $\phi 0.7$ is set $8 \mathrm{~mm}$ apart further. In the same way as the result of Fig.5, clear discrimination of the peaks was obtained. Fig.8 is the velocity profile by calculating the results of Fig.7. The smooth coincidence is obtained at switching points $(8 \mathrm{~mm}, 16 \mathrm{~mm})$ of measurement and the almost same results to that of Pitot-tube were obtained.

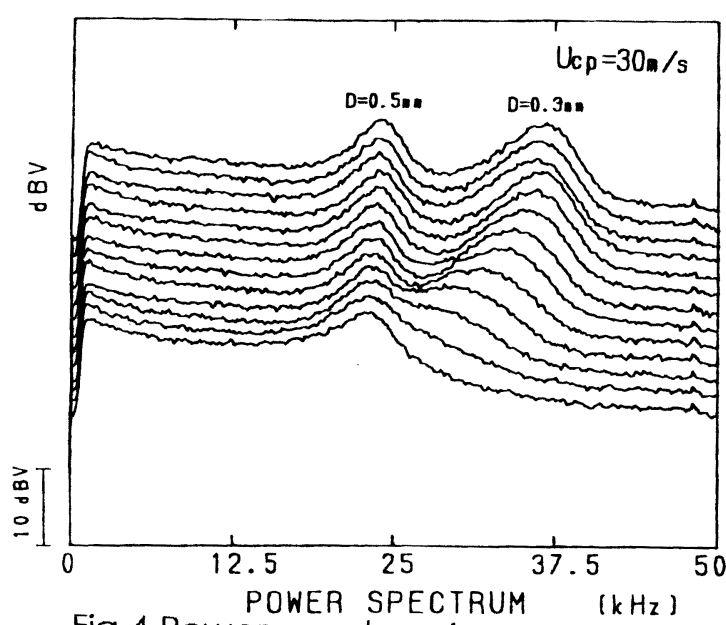
Distence from $x=0.13$ $x=1.14$
$x=2.15$
$x=3.16$
$x=5.17$ $x=3.16$
$x=4.17$
$x=5.19$ $x=5.18$
$x=5.19$
$x=7.20$
$x=5.21$ $x=7.20$
$x=8.21$
$x=0.22$ $x=8.21$
$x=9.22$
$x=10.23$ $x=10.23$
$x=11.24$
$x=12.25$

Fig. 4 Power spectru two generators ( $\phi 0.5-0.3$ )

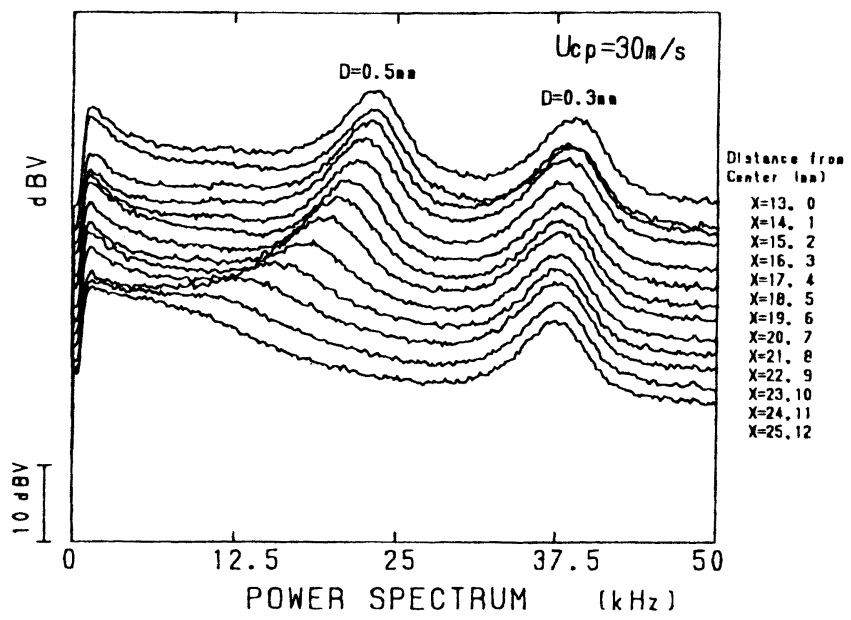

Fig. 5 Power spectrum In case of the two generators ( $\phi 0.3-0.5$ ) 

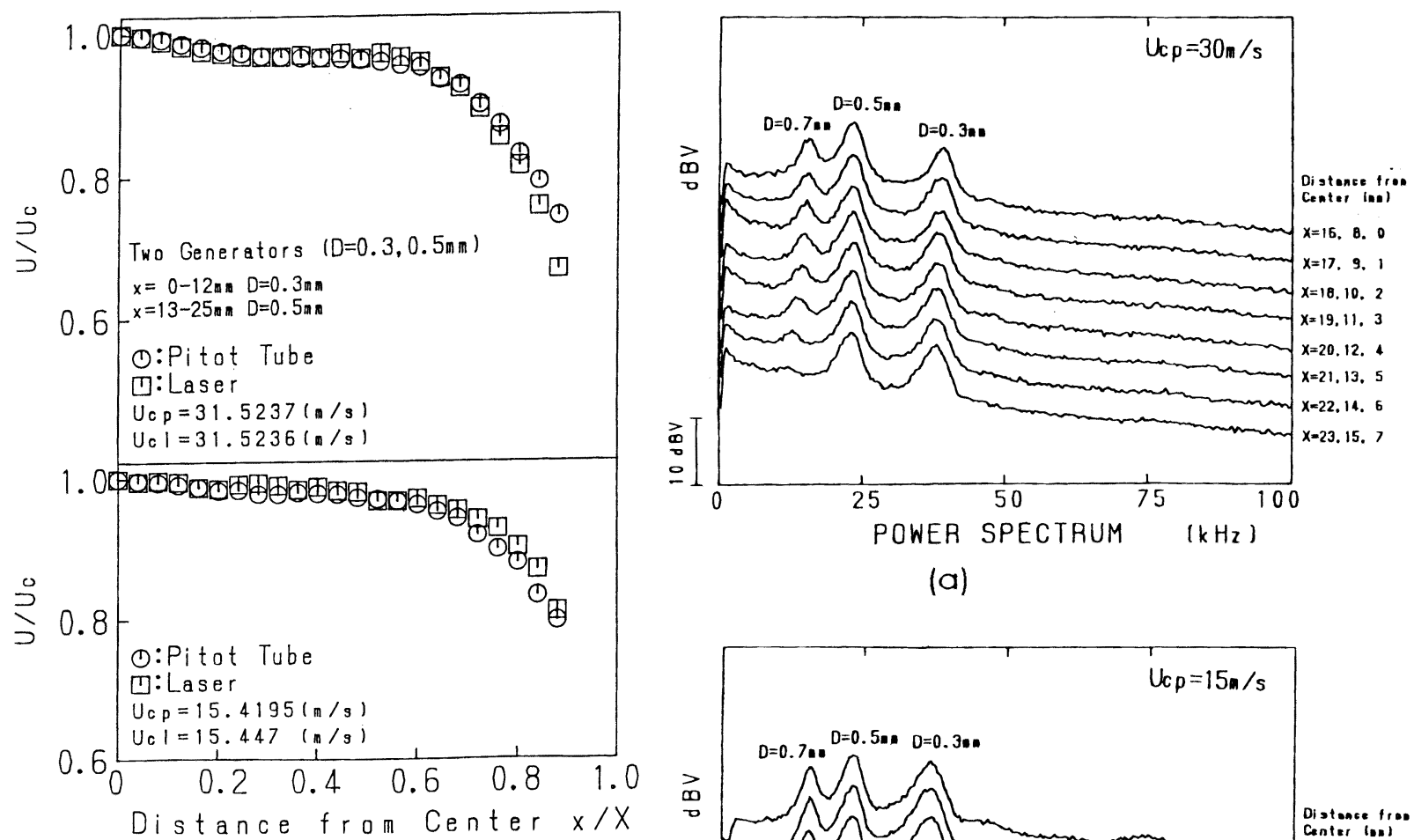

(a)

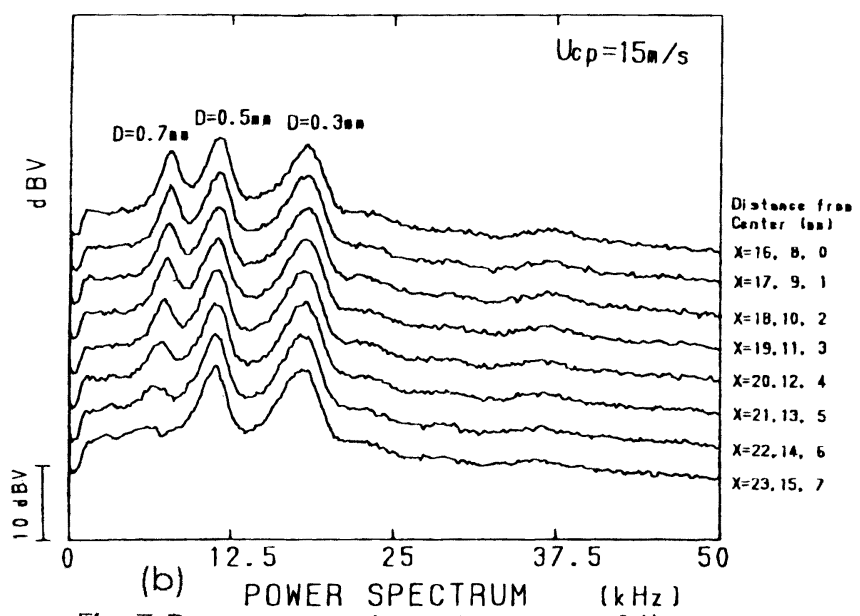

Fig.7 Power spectrum in case of the three generators

(B) The vortex generators of same size In case of the three generators stated previously, the cross sectional area of the generators over that of channel is about $3 \%$. Therefore it is difficult to increase the number of the generator further for the channel.

Then, in this method, the generators of further small diameter $(0.1 \mathrm{~mm})$ were utilized.

Nine generators are set at $0 \mathrm{~mm}, 5 \mathrm{~mm}, 10 \mathrm{~mm}$, $15 \mathrm{~mm}$ from the center of the channel and $1 \mathrm{~mm}$ apart each other from $19 \mathrm{~mm}$ to $23 \mathrm{~mm}$. This distance is wide enough not to interfere the Karman vortex each other because the distance is over 6 times of the diameter of the generator. The size $\phi 0.1$ makes possible the measurement of $1 \mathrm{~mm}$ interval.

The cross sectional area of the generators over the channel is about $1.8 \%$. 


\section{CONCLUSIONS}

Fig.9 shows the example of the power spectrums which were measured by this method. The power spectrum can be measured instantly by switching the electrical current as shown in Fig.9

Fig.10 shows the velocity profiles which were obtained by calculating the data of Fig.9. The results are good coincide with that of Pitot-tube.

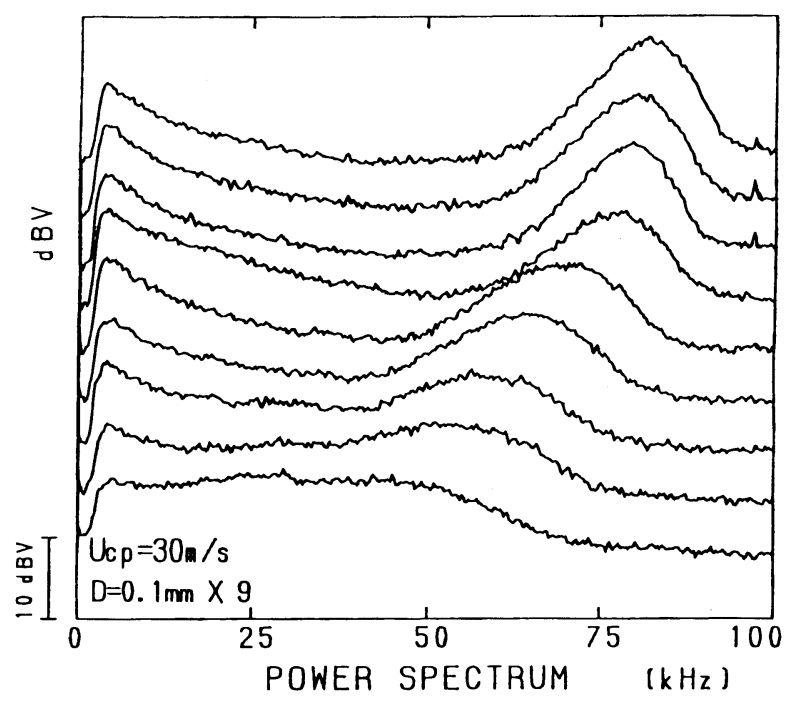

(a)

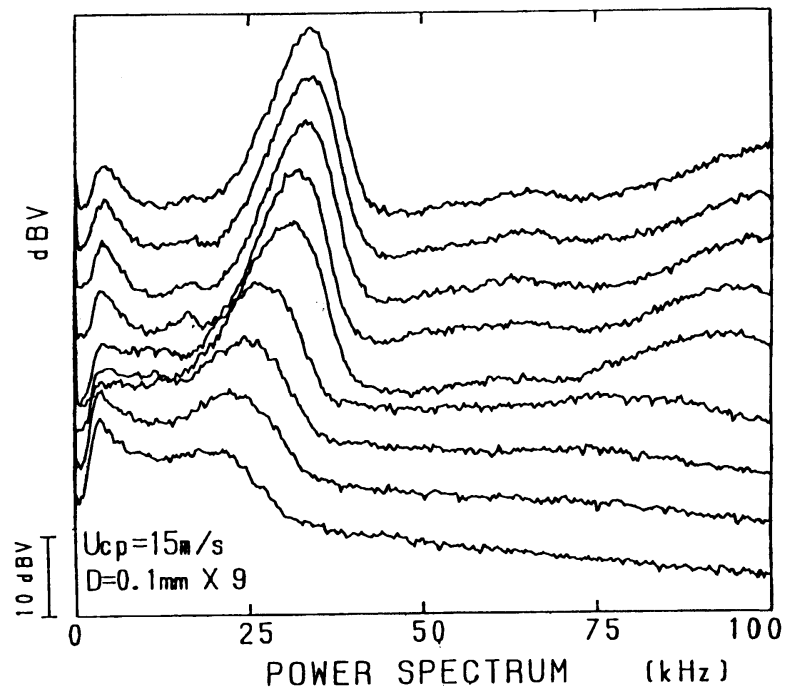

(b)

Fig.9 Power spectrum in case of

nine generators
(1) The simultaneous measurement of three point was available by using three vortex generators of different diameter.

(2) The measurement by the vortex generator of diameter as small as $\phi 0.1 \mathrm{~mm}$ is possible.

(3) By using nine generators of diameter of $\phi 0.1 \mathrm{~mm}$ and by switching the electrical charge which is applied to the generator, it was possible to change the measuring points instantly without moving the vortex generators or laser beam.

\section{REFERENCES}

1. Saito,S.,etal,Development of a Karman Vortex Velocimeter and a Flowmeter by Use of Laser Diode, FLUCOME 91 ASME 571-575 1991

2. Saito,S., Hashimoto,M., Wada,T., Development of Karman Vortex Flowmeter by Use of Laser Diode, FLOMEKO'93 ,335-340 1993

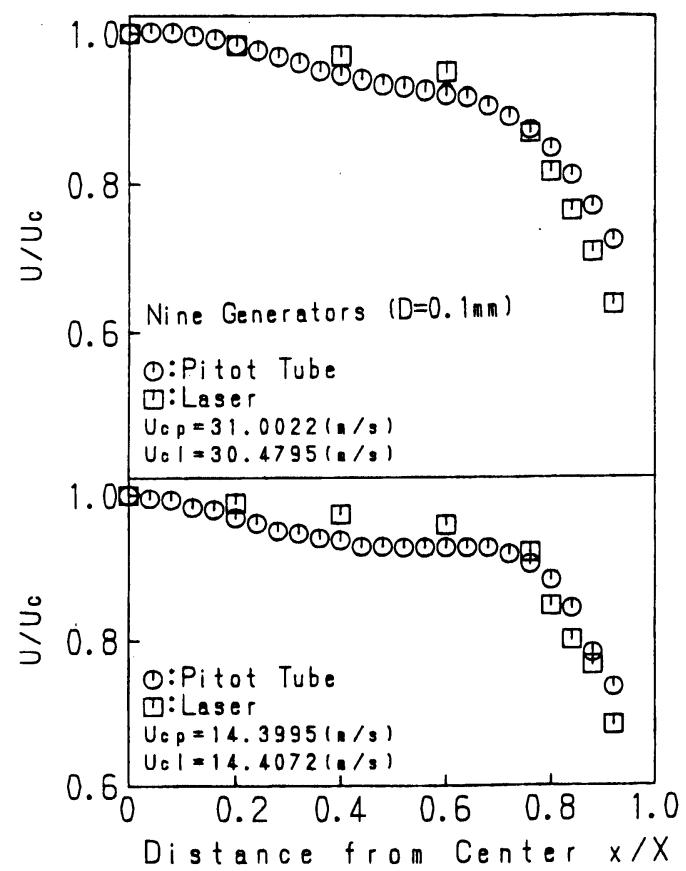

Fig. 10 Measured velocity profile 